\title{
Characteristic Function of Time-Inhomogeneous Lévy-Driven Ornstein-Uhlenbeck Processes
}

\author{
Frédéric Vrins \\ Louvain School of Management (LSM) \\ \& \\ Center for Operations Research and Econometrics (CORE) \\ Université catholique de Louvain \\ Chaussée de Binche 151, Office A.212, B-7000 Mons, Belgium \\ frederic.vrins@uclouvain.be
}

May 9, 2016

\begin{abstract}
Distributional properties -including Laplace transforms- of integrals of Markov processes received a lot of attention in the literature. In this paper, we complete existing results in several ways. First, we provide the analytical solution to the most general form of Gaussian processes (with nonstationary increments) solving a stochastic differential equation. We further derive the characteristic function of integrals of Lévy-processes and Lévy driven Ornstein-Uhlenbeck processes with timeinhomogeneous coefficients based on the characteristic exponent of the corresponding stochastic integral. This yields a two-dimensional integral which can be solved explicitly in a lot of cases. This applies to integrals of compound Poisson processes, whose characteristic function can then be obtained in a much easier way than using joint conditioning on jump times. Closed form expressions are given for gamma-distributed jump sizes as an example.
\end{abstract}

\section{Introduction}

Integrals of Markov processes are popular stochastic processes as a results of their numerous applications. Birth, death, bird-death and catastrophe processes in particular received a lot of attention in the context of queuing and storage problems [20] as well as in biology [19] or robotics [17]. From a broad perspective, general properties of integrals of Markov processes have been derived, like the time-evolution of associated moments [13]. With regards to the Laplace transforms in specific, closed form expressions have been derived in [22] for continuous-time Markov chains taking value on the set of positive integers. With regards to diffusion processes, very general results have been obtained for affine, quadratic and geometric models. The corresponding results are derived from the transition probabilities and the property that the infinitesimal generator is dependent on the space variable only, i.e the stochastic differential equations (SDE) has time-homogeneous coefficients. Explicit formulas for many standard processes including the integrated Orntein-Uhlenbeck (OU), Square-Root and Jacobi diffusions are available in [1],[15]. The specific case of integral of geometric Brownian motion, closely linked to squared Bessel processes, has extensively be studied by Yor [25], and a collection of papers on the topic is available [26].

In spite of this extensive literature, some important results are still lacking for interesting stochastic processes with sound applications. This is for instance the case of the generalized OU process, that is OU processes with time-varying coefficients and Lévy driving process. Similarly, to the best of our knowledge, there is no such results for compound Poisson processes.

In this paper, we are interested in the characteristic function (or equivalently, Laplace transform or moment-generating function -when it exists) of the path integral of the form

$$
\Lambda_{s, t}=\int_{s}^{t} \lambda_{u} d u
$$


where $\lambda=\left(\lambda_{t}\right)_{t \geqslant 0}$ is a Markov process on a probability space $\left(\Omega, \mathbb{F}, \mathcal{F}_{t}, \mathbb{Q}\right)$. We assume the sigma-field $\mathcal{F}_{t}$ contains all the information about $(\lambda, \Lambda)$ up to time $t$.

The paper is organized as follows. Section 2 is devoted to depict financial applications that will stress the importance of having the characteristic function available in closed form. We shall point out some processes $\lambda_{t}$ for which the integral is useful in this context. Associated characteristic functions will be derived in the remaining part of the paper. Most general results for Gaussian processes (not necessary with stationary increments) is addressed in Section 3. In Section 4, the corresponding results are obtained for Ornstein-Uhlenbeck processes with a background driving Lévy process (BDLP) which is not restricted to be a Brownian motion. Finally, Compound Poisson processes are investigated in Section 5 based on joint conditioning of jump times of the underlying Poisson process. Closed-form expressions are given when the jump sizes are Gamma-distributed.

\section{Financial motivation}

The most obvious example of the use of integrals of stochastic processes in finance is the pricing of Asian options, in which the payoff depends on the time average of the underlying stock (or index) price. When the underlying stock follows a geometric Brownian motion, as in the standard Black-Scholes case, this leads to the study of integrals of Squared Bessel processes and explains why this specific case received so much attention (see e.g. [12], [6]). In this context, Laplace transforms are interesting when explicit formula for the distributions are too involved or cannot be found. The corresponding distributions can be obtained by simple Fourier inversion, and all the moments are easily retreived by deriving the moment generating function. In some cases however, the explicit form of the characteristic function is very appealing in itself, as it direcly yields the calibration equation, as we now show.

Let $\mathbb{Q}$ stands for the risk-neutral measure and $\lambda_{t}$ for the risk-free short rate. Then, the time- $t$ price of a risk-free zero-coupon bond paying 1 unit of currency at time $T>t$ is

$$
B(t, T)=\mathbb{E}\left[\mathrm{e}^{-\Lambda_{t, T}} \mid \mathcal{F}_{t}\right]
$$

Consequently, the parameters of any given short rate process can be calibrated at time $t=0$ from the given yield curve $B(0, T)$ by making sure that the Laplace transform $\phi_{\Lambda_{T}}(x):=\mathbb{E}\left[\mathrm{e}^{-x \Lambda_{T}} \mid \mathcal{F}_{0}\right]$ of $\Lambda_{T}:=\Lambda_{0, T}$ satisfies

$$
B(0, T)=\phi_{\Lambda_{T}}(1)
$$

which is related to the characteristic function $\varphi_{\Lambda_{T}}(x)$ via $\varphi_{\Lambda_{T}}(x):=\phi_{\Lambda_{T}}(-i x)$.

For tractability reasons, the most popular short-rate models are, by far, Gaussian or square-root diffusion processes with constant coefficients, possibly shifted by a deterministic function. For simple short-rate models like Vasicek (with Ornstein-Uhlenbeck dynamics, OU) or Cox-Ingersoll-Ross (with square root diffusion dynamics, SRD), the analytical expression of the Laplace transform is available; see e.g. [5],[8],[9],[10]. However, the corresponding expressions for the Hull-White model, which is the extension of the Vasicek model with time-varying coefficients, is not available.

Expressions similar to 2.1 appear in credit risk modelling, in both reduced form and structural approaches (see e.g. [18],[4]). In reduced form (intensity) approaches, $\tau$ is modeled as the first jump of a Cox process $N_{t}$ where the stochastic intensity is given by a non-negative stochastic process $\lambda_{t}$ :

$$
\tau=\inf \left\{t: N_{t}>0\right\}
$$

Conditional on the path of the intensity $\lambda, N_{t}$ is a Poisson process, so that

$$
\mathbb{Q}(\tau>T)=\mathbb{Q}\left(N_{T}=0\right)=\mathbb{E}\left[\mathrm{e}^{-\int_{0}^{T} \lambda_{s} d s}\right]=\mathbb{E}\left[\mathrm{e}^{-\Lambda_{T}}\right]=\phi_{\Lambda_{T}}(1)
$$

The same form of survival probability is obtained in the so-called structural approach. In this setup, the default is modeled as the first passage time of an asset process below a liability threshold $K$ :

$$
\tau=\inf \left\{t: V_{t}<K\right\} \Leftrightarrow\left\{t: \mathrm{e}^{-V_{t}} \geqslant K^{\prime}\right\}
$$

The process $V_{t}$ is typically either a Brownian motion, a geometric Brownian motion or a subordinated process. The computation of the law of the first-passage times can be avoided by working in a specific 
framework where the survival probability takes the same form as in (2.4). Let $\Lambda_{t}$ be a non-decreasing grounded positive process and model the credit event as the first time where $\mathrm{e}^{-\Lambda_{t}}$ reaches a random threshold $U$ uniformly distributed in $[0,1]$. The passage time is almost surely unique, so that:

$$
\tau=\inf \left\{t: \mathrm{e}^{-\Lambda_{t}} \leqslant U\right\}=\left\{t: \mathrm{e}^{-\Lambda_{t}}=U\right\}
$$

Then, the survival probability $\mathbb{Q}(\tau>T)$ takes again the same form:

$$
\mathbb{Q}(\tau>T)=\mathbb{Q}\left(\mathrm{e}^{-\Lambda_{T}}>U\right)=\mathbb{E}\left[\mathbb{Q}\left(\mathrm{e}^{-\Lambda_{T}} \geqslant U\right)\right]=\mathbb{E}\left[\mathrm{e}^{-\Lambda_{T}}\right]=\phi_{\Lambda_{T}}(1)
$$

This suggests that instead of working with an intermediate intensity process, we could directly feed the default model with an non-decreasing Lévy process $\Lambda_{t}$. This setup has been used in [14] in the context of CDO pricing but in a purely numerical setup. The analytical expressions of the calibration equations are derived in [23]. The implied copulas form the class of so-called Sibuya copulas, which properties are studied in [24].

This setup, however is not appropriate when one desires to simulate survival probability curves $S(t, T)$. Indeed,

$$
\mathbb{Q}(\tau>T \mid \tau>t)=\mathbb{E}\left[\mathrm{e}^{-\left(\Lambda_{T}-\Lambda_{t}\right)} \mid \mathcal{F}_{t}\right]=\mathbb{E}\left[\mathrm{e}^{-\Lambda_{t, T}}\right]
$$

If the $\Lambda_{t}$ process is Lévy, the increments are independent of the past and if they are stationary, $\Lambda_{T}-\Lambda_{t} \sim$ $\Lambda_{T-t}$. Therefore, with $\delta=T-t$

$$
S(t, t+\delta):=\mathbb{Q}(\tau>t+\delta \mid \tau>t)=\mathbb{E}\left[\mathrm{e}^{-\Lambda_{\delta}}\right]=\phi_{\Lambda_{\delta}}(1)
$$

In other words, there is no memory effect. Producing different curves $S(t, t+\delta)$, depending on the path $\Lambda_{u}, u \in[0, t]$, can only be introduced by considering non-Lévy processes for $\Lambda_{t}$. One way to introduce such dependency is by considering $\Lambda_{t}$ as the integral of a underlying process $\lambda_{t}$, as in the intensity process.

\section{Gaussian processes}

As mentioned in the introduction, the expression of integrals of Gaussian processes are known in some specific cases, among which Vasicek (Ornstein-Uhlenbeck) is the most popular one. We generalise here previous results by working out the explicit form of the strong solutions for $\lambda_{t}$ and $\Lambda_{s, t}$ given $\lambda_{s}$ when $\lambda_{t}$ is the solution to the most general SDE associated to Gaussian processes.

It is very well-known that Gaussian process solving a stochastic differential equation (SDE) has dynamics of the form ${ }^{1}$

$$
d \lambda_{t}=\left(\alpha(t)-\beta(t) \lambda_{t}\right) d t+\sigma(t) d W_{t}, t \geqslant s, \quad \lambda_{s}
$$

where $W$ is a Brownian motion and $\alpha(t), \beta(t)$ and $\sigma(t)$ are deterministic integrable functions and $\lambda_{s}$ the initial condition. When the solution exists, it is possible to derive explicitly the strong solution for $\lambda_{t}$ and $\Lambda_{t}$ and obtain the corresponding characteristic function. We assume in this paper that the coefficients here are such that existence and uniqueness conditions are satisfied; see e.g. [16] or [21] for a detailed discussion.

Proposition 1 (General Gaussian Process). Consider eq.(3.1) and assume existence and uniqueness conditions are met. Suppose further that $\lambda_{s}$ is known at time $s \leqslant t$ and define

$$
\begin{aligned}
G(s, t):=e^{-\int_{s}^{t} \beta(u) d u} & , & I(s, t):=\int_{s}^{t} \alpha(u) G(u, s) d u \\
J(s, t):=\int_{s}^{t} \sigma(u) G(u, s) d W_{u} & , & K(s, t):=\int_{s}^{t} \sigma(s) G(s, u) d u
\end{aligned}
$$

Then, for any $t \geqslant s$, the solution to eq.(3.1) conditional upon $\lambda_{s}$ is

$$
\lambda_{t}=m(s, t)+G(s, t) J(s, t)
$$

\footnotetext{
${ }^{1}$ Not all Gaussian processes solve a SDE; this is for instance the case of fractional Brownian motion.
} 
which is Normally distributed with mean and variance respectively given by

$$
\begin{aligned}
m(s, t) & :=G(s, t)\left(\lambda_{s}+I(s, t)\right) \\
v(s, t) & :=G^{2}(s, t) \int_{s}^{t} \sigma^{2}(u) G^{2}(u, s) d u=\int_{s}^{t} \sigma^{2}(u) G^{2}(u, t) d u
\end{aligned}
$$

The integral $\Lambda_{s, t}$ is a shifted Ito integral

$$
\Lambda_{s, t}=M(s, t)+\int_{s}^{t} K(u, t) d W_{u}
$$

which is Normally distributed with mean and variance respectively given by

$$
\begin{aligned}
M(s, t) & :=(t-s) \lambda_{s}+\int_{s}^{t}(t-u)\left(\alpha(u)-\beta(u) G(s, u)\left(\lambda_{s}+I(s, u)\right)\right) d u \\
V(s, t) & :=\int_{s}^{t} K^{2}(u, t) d u
\end{aligned}
$$

Proof. See Appendix A.

Since $\Lambda_{s, t}$ is Gaussian with known mean and variance, the following corollary is obvious.

Corollary 3.1 (Laplace transform of $\Lambda_{s, t}$ ). The Laplace transform of $\Lambda_{s, t}$, conditional upon $\lambda_{s}$, is

$$
\phi_{\Lambda_{s, t}}(x)=\exp \left\{-M(s, t) x+\frac{x^{2} V(s, t)}{2}\right\}
$$

where $M(s, t), V(s, t)$ are given in Proposition 1.

Let us apply this result to get the expression and distribution of $\Lambda_{s, t}$ in some particular cases.

Example 3.1 (Integrated rescaled Brownian motion). Consider the case where $\lambda_{t}=\sigma W_{t}$.

Then,

$$
M(s, t)=(t-s) \lambda_{s}, K(s, t)=\sigma(t-s), V(s, t)=\frac{\sigma^{2}}{3}(t-s)^{3}
$$

This expression can be found directly using the stochastic version of Fubini's theorem on indicator functions.

Example 3.2 (Integrated OU process). The case where $\beta(t)$ and $\sigma(t)$ are constant is widely used in mathematical finance. It corresponds to $\alpha(t)=0, \beta(t)=\beta$ and $\sigma(t)=\sigma$ :

$$
\begin{aligned}
M(s, t) & =\frac{1-e^{-\beta(t-s)}}{\beta} \lambda_{s} \\
K(s, t) & =\frac{\sigma}{\beta}\left(1-e^{-\beta(t-s)}\right) \\
V(s, t) & =\left(\frac{\sigma}{2 \beta}\right)^{2}\left(2(t-s)+\frac{e^{-\beta(t-s)}}{\beta}\left(4-e^{-\beta(t-s)}\right)-\frac{6}{\beta}\right)
\end{aligned}
$$

The above examples correspond to special cases for which results exist (see e.g [5]). However, the above result allows to determine the Laplace transform of more general Gaussian processes, as shown in the examples below.

Example 3.3 (Prototypical swap exposure). Consider a Brownian bridge from 0 to $T$ rescaled by a constant $\sigma$ and shifted by the deterministic fontion $\gamma t(T-t)$. Such a process can be written as

$$
\lambda_{t}=(T-t)\left(\gamma t+\sigma \int_{0}^{t} \frac{1}{T-u} d W_{u}\right) \quad, 0 \leqslant s \leqslant t \leqslant T
$$

This process starts from 0 , its expectation follows the curve $\gamma t(T-t)$ and then goes back to 0 as $t \rightarrow T$. This is a Gaussian process with $\alpha(t)=\gamma(T-t), \beta(t)=\frac{1}{T-t}$ zero-mean and instantaneous volatility $\sigma(t)=\sigma$. Then

$$
M(s, T)=\lambda_{t} \frac{T-t}{2}+\frac{\gamma}{6}(T-t)^{3}, K(s, T)=\sigma \frac{T-s}{2}, V(s, T)=\frac{\sigma^{2}}{12}(T-s)^{3}
$$


See Appendix B for details.

Example 3.4 (Deterministically subordinated process). Consider the integral $\Lambda_{s, t}=\int_{s}^{t} f(s) W_{\theta(s)} d s$ where $\theta(s)$ is a strictly increasing and continuous function. Then,

$$
\Lambda_{s, t}=\int_{\theta(s)}^{\theta(t)} \frac{f \circ \theta^{-1}(u)}{\theta^{\prime}(u)} W_{u} d u
$$

corresponds to the integral of $\lambda_{t}:=\sigma(t) W_{t}$ where $\sigma(t):=\frac{f\left(\theta^{-1}(t)\right)}{\theta^{\prime}(t)}$. Its differential takes the form (3.1) where $\alpha(t):=0, \beta(t):=-\frac{\sigma^{\prime}(t)}{\sigma(t)}$ and instantaneous volatility $\sigma(t)$.

Consequently, provided that the existence conditions are met, $\Lambda_{s, t}$ is Normally distributed and the solution is given by (3.7) where the integration bounds are $s \leftarrow \theta(s), t \leftarrow \theta(t)$ and the parameters are defined above, and the characteristic function follows. ${ }^{2}$

\section{Lévy-driven Hull-White process}

In some cases, the characteristic function $\varphi_{\Lambda_{t}}(x)$ of the integrated process $\Lambda_{t}$ can be obtained by computing the characteristic function of a stochastic integral. This is in particular the case of generalized Lévy-driven OU processes (that is, where the random increments are controlled by a background driving Lévy process, BDLP), as we now show.

The log-characteristic function of a Lévy process $X_{t}$ takes the form

$$
\psi_{X_{t}}(x):=\ln \varphi_{X_{t}}(x)=t \ln \varphi_{X}(x)=: t \psi_{X}(x)
$$

where $\psi_{X}(x)$ is the characteristic exponent of the infinitely divisible distribution of $X:=X_{1}$ [7]. We can derive the $\log$-characteristic function of the stochastic integral $Y_{t}$ of a deterministic function $\sigma(t)$ with respect to a Lévy process $X-t$ as follows. Let $Y_{t}:=\int_{0}^{t} \sigma(s) d X_{s}$ where $\sigma(s)$ is integrable and $\left(X_{t}\right)_{t \geqslant 0}$ is Lévy processes with triplet $(\mu, \sigma, \nu)$ and $\nu(z)$ is the density of the Lévy measure. Then

$$
\begin{aligned}
& \psi_{X}(x)=i x \mu+\frac{1}{2} \sigma x^{2}+\int_{\mathbb{R}}\left(\mathrm{e}^{i x z}-1-i x z \mathbb{I}_{\{|z|<1\}}\right) \nu(d z) \\
& \psi_{Y_{t}}(x)=\ln \mathbb{E}\left[\mathrm{e}^{i x \int_{0}^{t} \sigma(s) d X_{s}}\right]
\end{aligned}
$$

Proposition 2 (Characteristic exponent of a stochastic integral with respect to a Lévy process). Let $\left(X_{t}\right)_{t \geqslant 0}$ be a Lévy process with characteristic exponent $\psi_{X}(x)$ and define the semimartingale $Y_{s, t}=$ $\int_{s}^{t} \sigma(u) d X_{u}$ for some deterministic integrable function $\sigma$. Then,

$$
\psi_{Y_{s, t}}(x)=\int_{s}^{t} \psi_{X}(\sigma(u) x) d u
$$

where $\psi_{Y_{s, t}}(x):=\ln \varphi_{Y_{s, t}}(x)$.

Proof. See Appendix C

The Lévy driven Hull-White process is defined as a Ornstein-Uhlenbeck process with time-varying coefficients and BDLP.

Proposition 3 (Characteristic exponent of integrated BDLP Hull-White process). Let $\left(\lambda_{t}\right)_{t \geqslant 0}$ be a Hull-White process driven by the Lévy process $X_{t}$ solution of the $S D E$

$$
d \lambda_{t}=\left(\alpha(t)-\beta(t) \lambda_{t}\right) d t+\sigma(t) d X_{t}
$$

Then, setting $G(s, t):=e^{-\int_{s}^{t} \beta(u) d u}$ as before,

$$
\psi_{\Lambda_{s, t}}(x)=i x M(s, t)+\int_{s}^{t} \psi_{X}\left(x \int_{u}^{t} \sigma(u) G(u, v) d v\right) d u
$$

\footnotetext{
${ }^{2}$ Observe that when $\theta(t)$ is not strictly increasing (i.e. mereley non-decreasing) and/or discontinuous, the corresponding characteristic function can be recovered by splitting the integral into pieces where the inveres of $\theta(s)$ exists, where $\theta(s)$ is constant and at time points where $\theta(t)$ jumps.
} 
Proof. Using the integration by parts technique used in the proof of Proposition 1, the solution $\Lambda_{s, t}$ is proven to be the same as in $(3.7)$ where $W_{t}$ is replaced by $X_{t}$ :

$$
\Lambda_{s, t}=M(s, t)+\int_{s}^{t} K(u, t) d X_{u}
$$

Therefore,

$$
\psi_{\Lambda_{s, t}}(x)=i x M(s, t)+\psi_{\int_{s}^{t} K(u, t) d X_{u}}(x)
$$

and the claim follows from Proposition 2 and the expression of $K(s, t)$ in Proposition 1.

Observe that SDE (4.5) is not the most general SDE with linear drift $\alpha(t)-\beta(t) x$ since the way jumps are handled corresponds to the special case where a jump of $X$ at $t$ of size $z$ triggers a jump of size $\sigma(t) z$ at $t$ for $\lambda_{t}$. We could perfectly want to have a jump $\lambda_{t}-\lambda_{t_{-}}$which does depend on $\left(\lambda_{t}, z\right)$ in a non-linear way (see e.g. [3] and [2] for a more advanced discussion and conditions on the coefficients $\alpha(t), \beta(t), \sigma(t)$ and the functional parameters of $X_{t}$ for existence and pathwise uniqueness).

Example 4.1 (OU driven by a Brownian Motion). We recover the characteristic function of the standard OU model driven by a Brownian motion from the strong solution

$$
\Lambda_{s, t}=M(s, t)+\int_{s}^{t} K(u, t) d W_{u}
$$

where $K(u, t)=\frac{\sigma}{\beta}\left(1-e^{-\beta(t-u)}\right)$. Since here $X=W$, the Lévy triplet is $(0, \sigma, 0)$ and the characteristic exponent reduces to $\psi_{X}(x)=-x^{2} / 2$. So,

$$
\int_{s}^{t} \psi_{X}(K(u, t) x) d u=-\int_{s}^{t} \frac{(x K(u, t))^{2}}{2} d u=-\frac{x^{2}}{2} V(s, t)
$$

Therefore,

$$
\psi_{\Lambda_{s, t}}(x)=i x M(s, t)-\frac{x^{2}}{2} V(s, t)
$$

in line with $\ln \phi_{\Lambda_{s, t}}(-i x)$ in eq. (3.10).

Let us apply this method to determine the characteristic function of $\Lambda_{t}$ where $\lambda_{t}$ is a non-Gaussian OU process driven by a gamma process $\gamma_{t}$.

Example 4.2 (OU driven by a gamma process). Consider the SDE

$$
d \lambda_{t}=-\beta \lambda_{t} d t+d \gamma_{t}
$$

with non-negative solution $\lambda_{t}=\left(\lambda_{s}+\int_{s}^{t} e^{-\beta(s-u)} d \gamma_{u}\right) e^{-\beta(t-s)}$ and the integral is

$$
\Lambda_{s, t}=\lambda_{s} \frac{1-e^{-\beta(t-s)}}{\beta}+\int_{s}^{t} \frac{1-e^{-\beta(t-u)}}{\beta} d \gamma_{u}
$$

The integrated process $\Lambda_{t}$ is a deterministc constant plus a stochastic integral of a deterministic function $\sigma(u)$ with respect to a Lévy process. Therefore,

$$
\psi_{\Lambda_{t}}(x)=i x \lambda_{s} \frac{1-e^{-\beta(t-s)}}{\beta}+\int_{s}^{t} \psi_{\gamma}\left(\frac{1-e^{-\beta(t-u)}}{\beta} x\right) d u
$$

where $\psi_{\gamma}(x)=\psi_{\gamma}(x ; \kappa, \alpha):=\ln \left(\frac{\kappa}{\kappa-i x}\right)^{\alpha}$ is the characteristic exponent of the gamma distribution driving the jump sizes. The integral can be written in terms of the dilogarithmic function $\mathrm{Li}_{2}(x)$

$$
(t-s) \psi_{\gamma}(x / \beta)+\frac{\alpha}{\beta}\left(L i_{2}(v)-L i_{2}\left(v e^{-\beta(t-s)}\right)\right)
$$

where $v=-\frac{i x}{\kappa \beta-i x}$. 
A similar approach has been used in $[11]^{3}$ to evaluate a joint characteristic function in the $T$-forward measure of a triple of processes which dynamics are given in the risk-neutral measure.

The following result is a straight consequence of Proposition 3.

Corollary 4.1 (Characteristic exponent of an integrated Lévy process). Let $\left(\lambda_{t}\right)_{t \geqslant 0}$ be a Lévy process. Then,

$$
\psi_{\Lambda_{s, t}}(x)=i x \lambda_{s}(t-s)+\int_{s}^{t} \psi_{\lambda}(x(t-u)) d u
$$

Example 4.3 (Stochastically subordinated Brownian motion). We are interested in the integral of $\lambda_{t}:=W_{X_{t}}$ that is, of the integral of a time-changed Brownian motion. Since for every Lévy process $\left(X_{t}\right)_{t \geqslant 0}$ we have $\psi_{X_{t}}(x)=t \psi_{X}(x)$,

$$
\varphi_{\lambda_{t}}(x)=\mathbb{E}\left[e^{i x \lambda_{t}}\right]=\mathbb{E}\left[\left(e^{i x W}\right)^{X_{t}}\right]=\mathbb{E}\left[e^{X_{t} \psi_{W}(x)}\right]=\mathbb{E}\left[e^{i X_{t}\left(-i \psi_{W}(x)\right)}\right]=\varphi_{X}^{t}\left(-i \psi_{W}(x)\right)
$$

or equivalently in terms of the characteristic exponent,

$$
\psi_{\lambda}(x)=\psi_{X}\left(-i \psi_{W}(x)\right)=\psi_{X}\left(i x^{2} / 2\right)
$$

In the case of a Gamma subordinator $X_{t}=\gamma_{t}$ with parameters $(\kappa, \alpha)$, the subordinated process is willknown to be a variance-gamma (Lévy) process. Indeed, $\lambda_{t}$ has the same distribution as the difference of two independent gamma processes, say $\gamma_{t}^{(a)}$ and $\gamma_{t}^{(b)}$, each with same parameters $(\alpha, \sqrt{2 \kappa})$. This can be seen by noting from the above result that $\varphi_{\lambda_{t}}(x)=\varphi_{\gamma}^{t}\left(i x^{2} / 2\right)$ :

$$
\phi_{\lambda_{t}}(x)=\left(\frac{2 \kappa}{2 \kappa+x^{2}}\right)^{\alpha t}=\left(\frac{\sqrt{2 \kappa}}{\sqrt{2 \kappa}+i x}\right)^{\alpha t}\left(\frac{\sqrt{2 \kappa}}{\sqrt{2 \kappa}-i x}\right)^{\alpha t}
$$

The log-characteristic function of the integral $\Lambda_{s, t}$ of $\lambda_{u}$ is thus given by

$$
\begin{aligned}
\psi_{\Lambda_{t}}(x) & =i x \gamma_{s}(t-s)+\psi_{\int_{s}^{t} \gamma_{u} d u}(x)+\psi_{\int_{s}^{t} \gamma_{u} d u}(-x) \\
& =i x \gamma_{s}(t-s)+\int_{s}^{t} \psi_{\gamma}(x(t-u)) d u+\int_{s}^{t} \psi_{\gamma}(x(u-t)) d u
\end{aligned}
$$

and

$$
\int_{s}^{t} \psi_{\gamma}(x(t-u)) d u=\frac{\kappa}{i x} \frac{\psi_{\gamma}(x(t-s) ; \kappa, \alpha)}{\varphi_{\gamma}(x(t-s) ; \kappa, 1)}-\alpha(t-s)(1-\ln \kappa)
$$

\section{Compound Poisson processes}

In this section, we focus on the integral of jump processes where the jumps arrive according to a Poisson process $N=\left(N_{t}\right)_{t \geqslant 0}$ and the size of the $i$-th jump is given by the random variable $X_{i}$ which are mutually independent. We then specialize to the case of compound Poisson process where $X_{i} \sim X$. We compute the Laplace transform $\phi_{\Lambda_{t}}(x)$ for which the analytical expression can be found for some laws of $X$. This can be done in several ways. We adopt here on a joint conditioning on the number of jumps of $N$ by time $t$ and the corresponding jump times $\vec{T}=\left(T_{1}, \ldots, T_{N_{t}}\right)$.

\subsection{Generalized Compound Process}

The Laplace transform $\phi_{\Lambda_{t}}(x)$ of the integrated process $\Lambda_{t}=\int_{0}^{t} \lambda_{s} d s$ where $\lambda_{t}=\sum_{i=0}^{N_{t}} X_{i}$ is a generalized compound Poisson process is obtained as an infinite sum of multidimensional integrals.

Proposition 4. Let $N$ ba a Poisson process with intensity $\theta$ and $X_{1}, X_{2}, \ldots$ be a sequence of independent random variables. Then, the Laplace transform of the integrated process

$$
\Lambda_{t}=\int_{0}^{t} \sum_{i=1}^{N_{u}} X_{i} d u
$$

\footnotetext{
${ }^{3}$ We are grateful to D. Madan for providing us with this reference.
} 
is given by

$$
\phi_{\Lambda_{t}}(x)=e^{-\theta t}\left(1+\sum_{n=1}^{\infty} \theta^{n} \int_{t_{1}=0}^{t} \phi_{X_{1}}\left(x\left(t-t_{1}\right)\right) \ldots \int_{t_{n}=t_{n-1}}^{t} \phi_{X_{n}}\left(x\left(t-t_{n}\right)\right) d \vec{t}_{n}\right)
$$

Proof. The law of $N_{t}$ is that of a $\operatorname{Poisson}(\theta t)$ random variable, that is

$$
p_{N_{t}}(n)=\frac{(\theta t)^{n} \mathrm{e}^{-\theta t}}{n !}, \quad n \in\{0,1,2, \ldots\}
$$

Furthermore, the laws of the time arrival of the $i$-th jump, $T_{i}$, and the time $\Delta_{i}$ elapsed between jumps $i$ and $i+1$ are known

$$
\begin{aligned}
\Delta_{i+1}:=T_{i+1}-T_{i} & \sim \operatorname{Exp}(\theta)=\operatorname{Gamma}(1, \theta) \\
T_{i} & =\operatorname{Gamma}(i, \theta)
\end{aligned}
$$

where we have set $T_{0}:=0$. Let us further note $\vec{T}_{n}=\left(T_{1}, \ldots, T_{n}\right)$ and $\vec{t}_{n}=\left(t_{1}, \ldots, t_{n}\right)$. Then, due to the independence between the times separating two consecutive jumps, we compute the joint density $p_{\vec{T}}\left(\vec{t}_{n}\right)$ of the first $n$ jump times $\vec{T}_{n}$ :

$$
p_{\vec{T}_{n}}\left(\vec{t}_{n}\right)=\mathbb{I}_{\left\{\vec{t}_{n}\right\}} \prod_{i=1}^{n} \theta \mathrm{e}^{-\theta\left(t_{i}-t_{i-1}\right)}=\mathbb{I}_{\left\{\vec{t}_{n}\right\}} \theta^{n} \mathrm{e}^{-\theta t_{n}}
$$

where $t_{0}:=0$ and $\mathbb{I}_{\left\{\vec{t}_{n}\right\}}$ is 1 if $\vec{t}_{n}$ a non-decreasing sequence of nonnegative numbers and 0 otherwise.

We can then compute the joint density $p_{\vec{T}_{n}, N_{t}}\left(\vec{t}_{n}, n\right)$ of $\left(\vec{T}_{n}, N_{t}\right)$ at $\left(\vec{t}_{n}, n\right)$ using $\left\{N_{t}=n\right\} \Leftrightarrow\left\{T_{n} \leqslant\right.$ $\left.t, T_{n+1}>t\right\}$ as follows:

$$
\begin{aligned}
p_{\vec{T}_{n}, N_{t}}\left(\vec{t}_{n}, n\right) & =\mathbb{I}_{\left\{\vec{t}_{n}\right\}} \mathbb{Q}\left(\vec{T}_{n} \in d \vec{t}_{n}, T_{n} \leqslant t, T_{n+1}>t\right) \\
& =\mathbb{I}_{\left\{\vec{t}_{n}\right\}} \mathbb{I}_{\left\{t \geqslant t_{n}\right\}} \mathbb{Q}\left(\vec{T}_{n} \in d \vec{t}_{n}, T_{n+1}>t\right) \\
& =\mathbb{I}_{\left\{\vec{t}_{n}\right\}} \mathbb{I}_{\left\{t \geqslant t_{n}\right\}} \int_{t}^{\infty} p_{\vec{T}_{n+1}}\left(\vec{t}_{n}, s\right) d s \\
& =\mathbb{I}_{\left\{\vec{t}_{n}\right\}} \mathbb{I}_{\left\{t \geqslant t_{n}\right\}} \theta^{n} \mathrm{e}^{-\theta t}
\end{aligned}
$$

This expression depends on $\vec{t}_{n}$ only via the indicator function $\mathbb{I}_{\left\{\vec{t}_{n}\right\}} \mathbb{I}_{\left\{t \geqslant t_{n}\right\}}$. We can now compute the Laplace transform of $\Lambda_{t}$ by conditioning on $\left(\vec{T}_{n}, N_{t}\right)$ :

$$
\begin{aligned}
\phi_{\Lambda_{t}}(x) & =\mathbb{E}\left[\mathrm{e}^{-x \int_{0}^{t} \sum_{i=1}^{N_{u}} X_{i}} d u\right] \\
& =p_{N_{t}}(0)+\sum_{n=1}^{\infty} \mathbb{E}\left[\mathrm{e}^{-x \sum_{i=1}^{n} X_{i}\left(t-t_{i}\right)} \mid \vec{T}_{n}=\vec{t}_{n}, N_{t}=n\right] p_{\vec{T}_{n}, N_{t}}\left(\vec{t}_{n}, n\right) \\
& =p_{N_{t}}(0)+\sum_{n=1}^{\infty} \int_{t_{1}=0}^{\infty} \ldots \int_{t_{n}=0}^{\infty} \prod_{i=1}^{n} \phi_{X_{i}}\left(x\left(t-t_{i}\right)\right) p_{\vec{T}, N_{t}}\left(\vec{t}_{n}, n\right) d \vec{t}_{n} \\
& =\mathrm{e}^{-\theta t}+\sum_{n=1}^{\infty} \theta^{n} \mathrm{e}^{-\theta t} \int_{t_{1}=0}^{t} \ldots \int_{t_{n}=t_{n-1}}^{t} \prod_{i=1}^{n} \phi_{X_{i}}\left(x\left(t-t_{i}\right)\right) d \vec{t}_{n}
\end{aligned}
$$

This form is appealing for two reasons. First, only the product of the characteristic functions of the jump size variables appear in the multiple integral as $p_{\vec{T}_{n}, N_{t}}$ can be sent outside provided that we restrict the integration domain according to the indicator function $\mathbb{I}_{\left\{\vec{t}_{n}\right\}} \mathbb{I}_{\left\{t \geqslant t_{n}\right\}}$. Second, this multiple integral can be written as

$$
\phi_{\Lambda_{t}}(x)=\mathrm{e}^{-\theta t}+\mathrm{e}^{-\theta t} \sum_{n=1}^{\infty} \theta^{n} \underbrace{\int_{t_{1}=0}^{t} \phi_{X_{1}}\left(x\left(t-t_{1}\right)\right) \ldots \underbrace{\int_{t_{n}=t_{n-1}}^{t} \phi_{X_{n}}\left(x\left(t-t_{n}\right)\right.}_{:=I_{n}})}_{I_{1}} d \vec{t}_{n}
$$


Therefore, the multiple integral can be computed recursively: only the last characteristic function $\phi_{X_{n}}$ is involved in the last integral (noted $I_{n}$ ) which only depends on time via $\left(t_{n-1}, t\right)$. The previous integrals, $I_{n-k}$, involves the product of $\phi_{X_{n-k}}(t-s)$ and $I_{n-k}(s, t)$, which limits the complexity of the computation.

Observe that when $x=0, \phi_{X_{i}}\left(x\left(t-t_{i}\right)\right)=1$ and the multiple integral collapses $t^{n} / n !$, so that

$$
\sum_{n=1}^{\infty} \frac{(t \theta)^{n}}{n !}=\mathrm{e}^{t \theta}-1
$$

and $\phi_{\Lambda_{t}}(0)=\mathrm{e}^{-\theta t}\left(1+\mathrm{e}^{t \theta}-1\right)=1$ as it should.

Corollary 5.1. The Laplace transform of the integrated process

$$
\Lambda_{s, t}=\int_{s}^{t} \sum_{i=1}^{N_{u}} X_{i} d u
$$

is given by

$$
\phi_{\Lambda_{s, t}}(x)=e^{-x(t-s) \lambda_{s}} \phi_{\tilde{\Lambda}_{t-s}}(x)
$$

where $\phi_{\tilde{\Lambda}_{t}}(x)$ is as $\phi_{\Lambda_{t}}(x)$ in eq. (5.2) but with $X_{i} \leftarrow X_{N_{s}+i}$.

Proof. By definition,

$$
\int_{s}^{t} \sum_{i=1}^{N_{u}} X_{i} d u=(t-s) \lambda_{s}+\int_{s}^{t} \sum_{i=N_{s}+1}^{N_{u}} X_{i} d u=(t-s) \lambda_{s}+\int_{s}^{t} \sum_{i=1}^{N_{u}-N_{s}} X_{N_{s}+i} d u
$$

The Poisson process has stationary increments, so the distribution of $N_{u}-N_{s} \sim N_{u-s}$ and

$$
\int_{s}^{t} \sum_{i=1}^{N_{u}} X_{i} d u \sim(t-s) \lambda_{s}+\int_{0}^{t-s} \sum_{i=1}^{N_{v}} X_{N_{s}+i} d v
$$

leading to

$$
\mathbb{E}\left[\mathrm{e}^{-x \int_{s}^{t} \sum_{i=1}^{N u} X_{i} d u}\right]=\mathrm{e}^{-x(t-s) \lambda_{s}} \phi_{\tilde{\Lambda}_{t-s}}(x)
$$

\subsection{Compound Poisson process with Gamma jump sizes}

The above expression for the Generalized Compound Poisson process is appealing but is hard to solve as no recursive formula can be found for solving the multiple integral. Even in the case where the $X_{i} \sim \operatorname{Exp}\left(\lambda_{i}\right)$, the resulting integrals seems intractable. However, when the $X_{i}$ 's are iid, recursion formula can be found as shown below.

Beforehand, observe however that Compound Poisson processes being Lévy processes with characteristic exponent

$$
\psi_{\lambda}(x)=\int_{\mathbb{R}}\left(\mathrm{e}^{i x z}-1\right) \nu(d z)
$$

where $\nu(d x)=\theta f(d x)$ and $f$ is the jump size distribution we have, from Corollary 2 ,

$$
\psi_{\Lambda_{s, t}}(x)=i x \lambda_{s}(t-s)+\int_{s}^{t} \int_{-\infty}^{\infty}\left(\mathrm{e}^{i z x(t-u)}-1\right) \nu(d z) d v
$$

Solving this simple time-space integral leads the Laplace transform of $\Lambda_{s, t}$. This approach seems considerably simpler than computing the infinite sum of multiple integrals (of arbitrarily large dimension) in eq. 5.15 .

However, when $X_{i} \sim \operatorname{Gamma}(\kappa, \alpha)$, a recursion formula can be found and the infinite sum of multiple integrals can be solved, and we shall adopt this alternative methodology for the sake of illustration. The results obviously agree with those easily obtained by computing eq.(5.23) with $\nu(d z)=$ $\theta \frac{\kappa^{\alpha}}{\Gamma(\alpha)} z^{\alpha-1} \mathrm{e}^{-\kappa z} d z$. 
Example 5.1 (Compound Poisson process with exponentially-distributed jump sizes). Let $N_{t}$ be a Poisson process with intensity $\theta$ and $X_{i} \sim \operatorname{Exp}(\kappa)=\operatorname{Gamma}(\kappa, 1)$ be iid exponential variables with Laplace transform $\phi_{X}(x)=\phi_{\gamma}(x ; \kappa, 1)$. Given that $\phi_{\tilde{\Lambda}_{t-s}}(x)=\phi_{\Lambda_{t-s}}(x)$ in the case of iid $X_{i}$, it is enough to compute $\phi_{\tilde{\Lambda}_{t}}(x)$. Then, the integral $\Lambda_{t}$ of the compound Poisson process $\lambda_{t}:=\sum_{i=1}^{N_{t}} X_{i}$ is

$$
\phi_{\Lambda_{t}}(x)=\left(e^{-t} \phi_{\gamma}(x t ; \kappa,-\kappa / x)\right)^{\theta}
$$

To see this, notice that

$$
\int_{s=t_{n-1}}^{t} \phi_{\gamma}(x(t-s)) d s=-\frac{\kappa}{x}[\ln (\kappa+x(t-s))]_{t_{n-1}}^{t}=-\frac{\kappa}{x} \ln \phi_{\gamma}\left(x\left(t-t_{n-1}\right)\right)
$$

Integrating that in the previous integral, we get

$$
\begin{aligned}
\int_{s=t_{n-2}}^{t} \phi_{\gamma}(x(t-s)) \int_{u=s}^{t} \phi_{\gamma}(x(t-u)) d u d s & =-\frac{\kappa}{x} \int_{s=t_{n-2}}^{t} \phi_{\gamma}(x(t-s)) \ln \phi_{\gamma}(x(t-s)) d s \\
& =+\frac{\kappa}{x} \int_{u=0}^{t-t_{n-2}} \phi_{\gamma}(x u) \ln \phi_{\gamma}(x u) d u \\
& =\left(-\frac{\kappa}{x}\right)^{2} \int_{u=0}^{\phi_{\gamma}\left(x\left(t-t_{n-2}\right)\right)} \frac{\ln v}{v} d v \\
& =\frac{1}{2}\left(-\frac{\kappa \ln \phi_{\gamma}\left(x\left(t-t_{n-2}\right)\right)}{x}\right)^{2}
\end{aligned}
$$

Finally, using $\int x^{-1} \ln ^{n} x=\frac{\ln ^{n+1} x}{n+1} d x$ and $t_{0}=0$,

$$
\int_{t_{1}=0}^{t} \phi_{X_{1}}\left(x\left(t-t_{1}\right)\right) \ldots \int_{t_{n}=t_{n-1}}^{t} \phi_{X_{n}}\left(x\left(t-t_{n}\right)\right) d \vec{t}_{n}=\frac{1}{n !}\left(-\frac{\kappa \ln \phi_{\gamma}(x t)}{x}\right)^{n}
$$

Example 5.2 (Compound Poisson process with gamma-distributed jump sizes.). Let $X_{i} \sim \operatorname{Gamma}(\kappa, \alpha)$ be iid gamma random variables with Laplace transform $\phi_{X}(x)=\phi_{\gamma}(x ; \kappa, \alpha)$ for $x<\kappa$ and $\alpha>1$ (the case $\alpha=1$ corresponds to the exponential above). Then,

$$
\phi_{\Lambda_{t}}(x)=e^{-\theta\left(t-\frac{\kappa}{x(\alpha-1)} \phi_{\gamma}(x t ; \kappa, \alpha-1)\right)}
$$

The above expression can be found easily by noting that

$$
\int_{s=u}^{t} \phi_{\gamma}(x(t-s)) d s=\int_{s=u}^{t} \phi_{\gamma}^{\alpha}(x(t-s) ; \kappa, 1) d s=\frac{\kappa}{x(\alpha-1)} \phi_{\gamma}^{\alpha-1}(x(t-u) ; \kappa, 1)
$$

so that

$$
\begin{aligned}
\int_{t_{1}=0}^{t} \phi_{X_{1}}\left(x\left(t-t_{1}\right)\right) \cdots \int_{t_{n}=t_{n-1}}^{t} \phi_{X_{n}}\left(x\left(t-t_{n}\right)\right) d \vec{t}_{n} & =\frac{(\kappa / x)^{n} \phi_{\gamma}^{n(\alpha-1)}(x t ; \kappa, 1)}{\prod_{i=1}^{n} i(\alpha-1)} \\
& =\frac{\left(\kappa \phi_{\gamma}(x t ; \kappa, \alpha-1)\right)^{n}}{n !(x(\alpha-1))^{n}}
\end{aligned}
$$

\section{A Proof of Proposition 1}

It is easy to find the explicit expression of the strong solution $\lambda_{t}$ of eq. (3.1) with initial value $\lambda_{s}, s \leqslant t$. Setting,

$$
G(t, T):=\mathrm{e}^{-\int_{t}^{T} \beta(s) d s}=1 / G(T, t)
$$

we get

$$
\begin{aligned}
\lambda_{t} & =G(s, t)\left(\lambda_{s}+I(s, t)+J(s, t)\right) \\
I(s, t) & :=\int_{s}^{t} \alpha(u) G(u, s) d u \\
J(s, t) & :=\int_{s}^{t} \sigma(u) G(u, s) d W_{u}
\end{aligned}
$$


In particular, $\lambda_{t}$ given $\lambda_{s}$ is normally distributed with mean

$$
m(s, t)=G(s, t)\left(\lambda_{s}+I(s, t)\right)
$$

The variance is easily obtained by noting that $\mathbb{E}[J(s, t)]=0$ and using Ito isometry:

$$
v(s, t)=G^{2}(s, t) \int_{s}^{t} \sigma^{2}(u) \mathrm{e}^{\int_{s}^{u} 2 \beta(v) d v} d u=G^{2}(s, t) \int_{s}^{t}\left(\frac{\sigma(u)}{G(s, u)}\right)^{2} d u=\int_{s}^{t}(\sigma(u) G(u, t))^{2} d u
$$

One can then plug this expression in the integration by parts formula. The resulting expression is again a Ito integral with a deterministic offset:

$$
\Lambda_{t, T}=T \lambda_{T}-t \lambda_{t}-\int_{t}^{T} u d \lambda_{u}=(T-t) \lambda_{t}+\int_{t}^{T}(T-u) d \lambda_{u}
$$

Using eq. (3.1), the integral becomes

$$
\int_{t}^{T}(T-u) d \lambda_{u}=\int_{t}^{T}(T-u)\left(\alpha(u)-\beta(u) \lambda_{u}\right) d u+\int_{t}^{T}(T-u) \sigma(u) d W_{u}
$$

The first integral is

$$
\int_{t}^{T}(T-u)\left(\alpha(u)-\beta(u) G(t, u)\left(\lambda_{t}+I(t, u)\right)\right) d u-\int_{t}^{T}(T-u) \beta(u) G(t, u) J(t, u) d u
$$

All these integrals are deterministic integrals except the last one

$$
\begin{aligned}
& \int_{t}^{T}(T-u) \beta(u) G(t, u) \int_{t}^{u} \sigma(v) G(v, t) d W_{v} d u \\
= & \int_{t}^{T} \int_{t}^{u} \sigma(v) G(v, t) d W_{v} d_{u}\left(\int_{t}^{u}(T-s) \beta(s) G(t, s) d s\right) \\
= & \left(\int_{t}^{T} \sigma(v) G(v, t) d W_{v}\right)\left(\int_{t}^{T}(T-s) \beta(s) G(t, s) d s\right) \\
- & \int_{t}^{T} \sigma(u) G(u, t)\left(\int_{t}^{u}(T-s) \beta(s) G(t, s) d s\right) d W_{u} \\
= & \int_{t}^{T} \sigma(u) G(u, t)\left(\int_{u}^{T}(T-s) \beta(s) G(t, s) d s\right) d W_{u}
\end{aligned}
$$

So the expression for $\Lambda_{t, T}$ is the sum of deterministic functions

$$
M(t, T)=(T-t) \lambda_{t}+\int_{t}^{T}(T-u)\left(\alpha(u)-\beta(u) G(t, u)\left(\lambda_{t}+I(t, u)\right)\right) d u
$$

and stochastic integrals

$$
\begin{aligned}
S(t, T) & =\int_{t}^{T} \sigma(u)\left((T-u)+G(u, t) \int_{u}^{T}(s-T) \beta(s) G(t, s) d s\right) d W_{u} \\
& =\int_{t}^{T} \sigma(u)\left(\int_{u}^{T} G(u, s) d s\right) d W_{u} \\
& =\int_{t}^{T} K(u, T) d W_{u}
\end{aligned}
$$

setting $K(s, t):=\int_{s}^{t} \sigma(s) G(s, u) d u$.

The integral $\Lambda_{s, t}$ is Normally distributed with mean $M(s, t)$ given above and variance

$$
V(s, t)=\int_{s}^{t} K^{2}(u, t) d u
$$




\section{B Proof for Example 3.3}

It is trivial to see that

$$
G(t, u)=\frac{T-u}{T-t}, \quad K(t, T)=\sigma \int_{t}^{T} G(t, u) d u=\sigma \frac{T-t}{2}
$$

Furthermore,

$$
\begin{aligned}
M(t, T) & =(T-t) \lambda_{t}+\int_{t}^{T}(T-u)\left(\gamma(T-u)-\frac{1}{T-u} G(t, u)\left(\lambda_{t}+I(t, u)\right) d u\right. \\
& =(T-t) \lambda_{t}+\gamma \int_{t}^{T}(T-u)^{2} d u-\lambda_{t} \int_{t}^{T} G(t, u) d u-\int_{t}^{T} \int_{t}^{u} \gamma(T-v) G(v, u) d v d u \\
& =\lambda_{t} \frac{T-t}{2}+\frac{\gamma}{3}(T-t)^{3}-\gamma \int_{t}^{T} \int_{t}^{u}(T-u) d v d u \\
& =\lambda_{t} \frac{T-t}{2}+\frac{\gamma}{3}(T-t)^{3}-\gamma \int_{t}^{T}(T-u)(t-u) d u \\
& =\lambda_{t} \frac{T-t}{2}+\frac{\gamma}{3}(T-t)^{3}-\gamma \frac{(T-t)^{3}}{6} d u \\
& =\lambda_{t} \frac{T-t}{2}+\frac{\gamma}{6}(T-t)^{3}
\end{aligned}
$$




\section{Proof of Proposition 2}

We give the proof for $s=0$ but the development remains valide for any lower bound $0 \leqslant s \leqslant t$. Every Lévy process with Lévy measure $\nu$ can be written as (Prop. 3.7 Cont \& Tankov)

$$
X_{t}=\gamma t+\sigma W_{t}+\int_{0}^{t} \int_{\mathbb{R}} x J_{X}(d s \times d x)-\int_{0}^{t} \int_{|x| \leqslant 1} x \mu(d s \times d x)
$$

where $J_{X}(d s \times d x)$ is a Poisson random measure with intensity $\mu(d s \times d x)=\nu(d x) d s$. The differential of this Lévy process is

$$
d X_{s}=\gamma d s+\sigma d W_{s}+\int_{\mathbb{R}} x J_{X}(d s \times d x)-\int_{|x| \leqslant 1} x \mu(d s \times d x)
$$

So that for any integrable deterministic function $f(s)$, the stochastic integral $Y_{t}=\int_{0}^{t} f(s) d X_{s}$ takes the form

$$
Y_{t}=\gamma \int_{0}^{t} f(s) d s+\sigma \int_{0}^{t} f(s) d W_{s}+\int_{0}^{t} \int_{\mathbb{R}} x f(s) J_{X}(d s \times d x)-\int_{0}^{t} \int_{|x| \leqslant 1} x f(s) \nu(d x) d s
$$

The characteristic exponent of $X=X_{1}$ is

$$
\begin{aligned}
\psi_{X}(u) & =i \gamma u-\frac{\sigma^{2} u^{2}}{2}+\int_{\mathbb{R}}\left(\mathrm{e}^{i u x}-1\right) \nu(d x)-\int_{|x| \leqslant 1} i u x \nu(d x) \\
& =i \gamma u-\frac{\sigma^{2} u^{2}}{2}+\int_{\mathbb{R}}\left(\mathrm{e}^{i u x}-1-i u x \mathbb{1}_{\{|x| \leqslant 1\}}\right) \nu(d x)
\end{aligned}
$$

Therefore, the log-characteristic function $\psi_{Y_{t}}(u)=\ln \varphi_{Y_{t}}(u)$ of the stochastic integral $Y_{t}$ becomes

$$
\begin{aligned}
\psi_{Y_{t}}(u) & =\int_{0}^{t}\left(i \gamma(u f(s))-\frac{\sigma^{2}(u f(s))^{2}}{2}+\int_{\mathbb{R}}\left(e^{i x(u f(s))}-1-i x(u f(s)) \mathbb{I}_{\{|x| \leqslant 1\}}\right) \nu(d x)\right) d s \\
& =\int_{0}^{t} \psi_{X}(u f(s)) d s
\end{aligned}
$$

where we have used the exponential formula for Poisson random measure $\mathbb{E}\left[\mathrm{e}^{\int_{[0, T] \times B} g(s, x) J_{X}(d s \times d x)}\right]=$ $\mathrm{e}^{\int_{[0, T] \times B}\left(\mathrm{e}^{g(s, x)}-1\right) \mu(d s \times d x)} \cdot 4$

\section{Proof for Example 4.2}

Setting $x^{\prime}=i x$,

$$
\begin{aligned}
\psi_{\gamma}\left(\frac{1-\mathrm{e}^{-\beta(t-u)}}{\beta} x\right) & =\alpha \ln (\kappa \beta)-\alpha \ln \left(\kappa \beta-x^{\prime}+x^{\prime} \mathrm{e}^{-\beta(t-u)}\right) \\
& =\alpha \ln (\kappa \beta)-\alpha \ln \left(1+\frac{x^{\prime} \mathrm{e}^{-\beta(t-u)}}{\kappa \beta-x^{\prime}}\right)-\alpha \ln \left(\kappa \beta-x^{\prime}\right) \\
& =\psi_{\gamma}(x / \beta)-\alpha \ln \left(1+\frac{x^{\prime} \mathrm{e}^{-\beta(t-u)}}{\kappa \beta-x^{\prime}}\right)
\end{aligned}
$$

Set $v:=-\frac{x^{\prime} \mathrm{e}^{-\beta(t-u)}}{\kappa \beta-x^{\prime}}, v_{1}:=-\frac{x^{\prime} \mathrm{e}^{-\beta(t-s)}}{\kappa \beta-x^{\prime}}$ and $v_{2}:=-\frac{x^{\prime}}{\kappa \beta-x^{\prime}}$

$$
\begin{aligned}
\int_{s}^{t} \psi_{\gamma}\left(\frac{1-\mathrm{e}^{-\beta(t-u)}}{\beta} x\right) d u & =(t-s) \psi_{\gamma}(x / \beta)-\alpha \int_{s}^{t} \ln \left(1+\frac{x^{\prime} \mathrm{e}^{-\beta(t-u)}}{\kappa \beta-x^{\prime}}\right) d u \\
& =(t-s) \psi_{\gamma}(x / \beta)+\frac{\alpha}{\beta} \int_{v_{1}}^{v_{2}} \frac{\ln (1-v)}{v} d v \\
& =(t-s) \psi_{\gamma}(x / \beta)+\frac{\alpha}{\beta}\left(L i_{2}\left(v_{2}\right)-L i_{2}\left(v_{1}\right)\right)
\end{aligned}
$$

\footnotetext{
${ }^{4}$ We thank P. Tankov for discussions on this formula when the integral is jointly taken over the time-space set.
} 


\section{References}

[1] C. Albanese and S. Lawi. Laplace transforms of integrals of Markov processes. Markov Processes and Related Fields, 11:677-724, 2005.

[2] O. Barndorff-Nielsen and N. Shepard. Non-Gaussian Ornstein-Uhlenbeck-based models and some of their uses in financial economics. Journal of the Royal Statistical Association, 63(2):167-241, 2001.

[3] R. Bass. Stochastic differential equations with jumps. Probability Surveys, 1:1-19, 2004.

[4] T. Bielecki, M. Jeanblanc, and M. Rutkowski. Credit risk modeling. Technical report, Osaka (Japan), 2011.

[5] D. Brigo and F. Mercurio. Interest Rate Models - Theory and Practice. Springer, 2006.

[6] P. Carr and M. Schroder. Bessel processes, the integral of Geometric Brownian Motion and Asian options. Theory of Probability and Applications, 48(3):400-425, 2004.

[7] R. Cont and P. Tankov. Financial Modelling with Jump Processes. Chapman \& Hall, 2004.

[8] JC. Cox, J.E. Ingersoll, and S.A. Ross. An intertemporal general equilibrium model of asset prices. Econometrica, 53:363-384, 1985.

[9] JC. Cox, J.E. Ingersoll, and S.A. Ross. A theory of the term structure of interest rates. Econometrica, 53:385-407, 1985.

[10] D. Dufresne. The integrated square-root process. Technical report, 2001.

[11] E. Eberlein, D. Madan, M. Pisotrius, and M. Yor. A simple stochastic rate model for rate equity hybrid products. Technical report, Dpt Mathematical Stochastics, University of Freiburg, 2013.

[12] H. Geman and M. Yor. Bessel processes, Asian options and perpetuities. Mathematical Finance, 3:349-75, 1993 .

[13] D.T. Gillespie. Markov Processes: An Introduction for Physical Scientists. Academic Press, 1992.

[14] J. Hull and A. White. Dynamic models of portfolio credit risk : A simplified approach. Journal of Derivatives, 15(4):9-28, 2008.

[15] T. Hurd and A. Kuznetsov. Explicit formulas for Laplace transforms of stochastic integrals. Markov Processes and Related Fields, 14:277-290, 2008.

[16] I. Karatzas and S. Shreve. Brownian Motion and Stochastic Calculus. Springer, 2005.

[17] Y. Khaluf, M. Pace, Rammig F., and M. Dorigo. Integrals of markov processes with applications in swarm robotics modelling. Technical report, 2012.

[18] D. Lando. Credit Risk Modeling: Theory and Applications. Pinceton Series in Finance. Pinceton University Press, 2004.

[19] M. Mangel and C. Tier. Four facts every conservation biologists should know about persistence. Ecology, 73(3):607614, 1994.

[20] P. Moran. The Theory of Storage. Methuen Ltd, 1959.

[21] B. Øksendal. Stochastic Differential Equations. An Introduction with Applications. Universitext. Springer, 2003.

[22] P. Pollett and V. Stefanov. Path integrals for continuous-time Markov chains. Journal of Applied Probability, 39(4):901-904, 2002.

[23] F. Vrins. Analytical pricing of basket default swaps in a dynamic hull-white framework. Journal of Credit Risk, 6(4):85-111, 2011. 
[24] F. Vrins and M. Hofert. Sibuya copulas. Journal of Multivariate Analysis, 114:318-337, 2013.

[25] M. Yor. On some exponential functionals of Brownian motion. Advances in Applied Probability, 24(3):509-531, 1992.

[26] M. Yor. Exponential Functionals of Brownian Motion and Related Processes. Springer-Verlag, 2001. 